\title{
The Value of FLT3, NPM1 and DNMT3A Gene Mutation Analysis in Acute Myeloid Leukemia Diagnosis
}

\author{
Claudia Bănescul ${ }^{1 *}$, Cristina Skrypnyk ${ }^{2}$ \\ 1. Laboratory of Molecular Biology/Genetics Center for Advanced Medical and Pharmaceutical \\ Research, University of Medicine, Pharmacy, Sciences and Technology of Târgu Mureş, Romania \\ 2. Arabian Gulf University, College of Medicine, Molecular Medicine Department, Al Jawhara Center \\ for Molecular Medicine, Manama, Bahrain
}

Received: 25 $5^{\text {th }}$ March 2019; Accepted: $7^{\text {th }}$ April 2019; Published: $2^{\text {nd }}$ July 2019

Myeloid neoplasms, especially acute myeloid leukemia (AML), are characterized by abnormal proliferation of hematopoietic cells and by genomic instability with a high diversity of chromosomal and molecular abnormalities $(1,2)$. The 2016 revised World Health Organization classification of AML and myeloid neoplasm emphasized the value of molecular genetics testing in AML risk stratification and prognosis, especially for patients with a normal karyotype $(3,4)$. The gene mutation data are adding an important contribution to the immunophenotype and the clinical features in defining clinicopathological relevant disease entities $(3,5)$. The investigation of the genome profile in AML has fundamentally changed the approach of AML patients. AML patients are routinely investigated for the presence of mutations in FMS-related tyrosine kinase 3 (FLT3) and NPM1 genes. Recently some studies recommended also the DNA methyltransferase 3A (DNMT3A) gene mutation analysis at the AML diagnosis time (6-8). Roloff et al. sug- gested that AML cases have on average three acquired mutations at the diagnostic although the European LeukemiaNet (ELN) 2017 prognostic model considers only one gene mutation in the most of AML cases and two in a few cases (9).

\section{FLT3 gene mutation}

FLT3 mutations are common molecular abnormalities in AML (10) and have therefore proven to be a target for FLT3 tyrosine kinase inhibitors (FLT3 TKIs) enabling the personalized treatment of AML. FLT3 internal tandem duplications (ITD) were reported in $30 \%$ of AML patients and the tyrosine kinase domain (TKD) point mutations detected in about $5 \%$ of AML cases. The prognostic significance of FLT3 ITD is influenced by allelic ratio (FLT3 ITD to FLT3 wild-type). According to the 2017 ELN recommendations, a high allelic ratio is considered to be greater than 0.5 (FLT3 ITD high; >0.5) while a low allelic ratio is considered less than

\footnotetext{
*Corresponding author: Claudia Bănescu, Genetics Laboratory, Center for Advanced Medical and Pharmaceutical Research, University of Medicine, Pharmacy, Science and Technology of Târgu Mureș, 38 Gh Marinescu St, 540139, Tirgu Mures, Romania. E-mail: claudia.banescu@gmail.com
} 
0.5 (FLT3 ITD low; <0.5) (11). Mutation within FLT3 gene are common in AML cases with normal karyotype but they are also associated with cytogenetic aberrations, such as $\mathrm{t}(15 ; 17) /$ $P M L-R A R A$, core binding factor-AML $(C B F$ AML) $\mathrm{t}(6 ; 9)$ and DEK-NUP214 abnormalities (12). FLT3 mutation testing is recommended in all AML patients in parallel with cytogenetic analyses as finding a FLT3 gene mutation is a negative prognostic marker and Midostaurin, a FLT3 TKI targeted therapy, is available (12). In addition, FLT3 gene mutation may be used as a predictor for relapse in AML, had a negative effect on survival time (13), and is frequent in older patients (14). Considering the fact that FLT3ITD is acquired late in leukemogenesis and that FLT3-ITD mutation may be lost at the time of relapse, it is not recommended to use FLT3 mutation as a marker for minimal residual disease (MRD) monitoring (9).

\section{NPM1 gene mutation}

NPM1 mutations are the commonest AML mutations identified up to now in adult patients, occurring in about $30 \%$ of cases, including half of cytogenetically normal AML patients and it is associated with a good prognosis in the absence of FLT3 ITD mutation $(14,15)$. NPM1 mutations were almost always found in association with co-occurrence mutations and reported usually as late driver secondary events (8). Papaemmanuil et al. reported that the most frequently observed co-mutations in AML patients with a NPM1 mutation were DNMT3A (54\%), FLT3 ITD (39\%), NRAS (19\%), TET2 (16\%) and PTPN11 (15\%) (8). The same study observed that co-occurrence of mutations in NPM1, FLT3 ITD, and DNMT3A predicted an especially adverse prognosis of the young AML patients (8). Recently, the study performed by Patel et al indicated that a high NPM1 mutant allele at diagnosis in de novo AML is an independent predictor of unfavorable clinical outcomes, particularly in patients treated with stem-cell transplant and in patients with DNMT3A co-mutation (16). The prognostic effect of NPM1 mutation in de novo AML may be influenced by the mutated allele burden (16). Other studies reported also that co-occurrance of NPM1 mutations with other common gene mutations in AML patients is associated with a poor prognosis (17). NPM1 mutations are considered to be mutually exclusive with other genomic rearrangements and/or chromosomal aneuploidy in AML patients. It is estimated that $75 \%$ of $N P M 1$-mutated AML associate mutations in DNA hydroxymethylation genes [such as DNMT3A, Ten-Eleven Translocation 2 (TET2), isocitrate dehydrogenase genes IDH1 and IDH2)] (18).

Furthermore, NPMI gene mutation has been validated as a good marker for assessment of minimal residual disease and as a predictor for AML relapse (9).

\section{DNMT3A gene mutation}

DNMT3A gene mutation are found in about $25 \%$ $30 \%$ of all AML cases, the most of them are 1ocalized in R882 domain and are more frequent in older AML cases. Mutations in DNMT3A gene are associated with poor prognosis and with a higher relapse rate (4). Recently it was reported that in the absence of high-risk cytogenetics, DNMT3A mutation status has a negative impact on prognostic outcome in the presence or absence of FLT3 and/or NPM1 mutation $(6,14)$. DNMT3A mutation is an important predictor of shorter overall survival (OS) in patients diagnosed with AML, especially in those with normal karyotype or intermediate-risk cytogenetics. The OS of the AML cases with DNMT3A mutation was reported by Kumar et al. to be poorer than that of AML patients with NPM1 mutation but was better than that of the AML cases with FLT3 ITD mutation (6). About $80 \%$ of AML 
patients harboring DNMT3A mutation had also NPM1 mutation (19).

A recent study revealed that $\mathrm{R} 882$ mutations in DNMT3A gene were associated with an increased number of white blood cells (WBC) and a higher percentage of blasts at the moment of diagnosis, with M4-M5 AML subtype according to French-American-British (FAB) classification and with normal karyotype (20). The same study observed that the mutation burden decreased after allogeneic hematopoietic stem cell transplantation (alloHSCT) and suggested it as the optimal therapy choice for the eradication of DNMT3A R882 mutation in AML cases (20). Recently it was observed that $D N M T 3 A$ is one of the most frequently commutated gene with isocitrate dehydrogenase-2 (IDH2) in relapsed or refractory AML cases (21).

\section{FLT3 ITD mutation in association with NPM1 mutation}

The relapse rate and overall survival in AML patients with FLT3 ITD mutation is influenced by the ITD allelic ratio. It was reported that patients with NPM1 mutation and FLT3 ITD mutation but with a low allelic ratio $(<0.5)$ have a similar outcome (favorable prognosis) as patients with a NPM1 mutation but without FLT3 ITD; therefore, both groups are now considered favorable (and added to favorable risk group according to ELN 2017 recommendations) (11). Contrariwise, AML with wild-type NPMI and FLT3 ITD with a high allelic ratio $(>0.5)$ has an adverse prognosis and is included in the adverse-risk group (11).

\section{FLT3 ITD mutation in association with DNMT3A R882}

Recently, it was reported by Tang et al. that AML patients with FLT3 ITD and DNMT3A R882 double mutation had a poor prognosis, and that FLT3 ITD+ and DNMT3A R882+ double muta- tion was an independent factor for poor outcome post-transplantation (7).

Another recent study performed by Ardestani et al. revealed that patients with both $D N M T 3 A$ R882 and FLT3 ITD mutations had the worst OS and relapse-free survival compared with AML cases with one mutation (22). The OS at 5-year was $0 \%$ for AML patients with FLT3 ITD + DNMT3A R882+ double mutation versus those with no DNMT3A R882 or FLT3 ITD mutation $(62 \%)$. Based on their findings, presence of FLT3 ITD+ and DNMT3A R882+ double mutations represent an unfavorable prognostic factor in AML patients even after allogeneic hematopoietic stem cell transplantation (22). Recently it was reported that FLT3 ITD+ and DNMT3A $\mathrm{R} 882+$ double mutation is significantly associated with a lower $\operatorname{OS}(\mathrm{P}=0.016)$ and it has a significant negative effect on complete remission rates (14).

\section{NPM1, DNMT3A and FLT3 combined mutations}

NPM1, DNMT3A, and FLT3 ITD combined mutated genotype represented the most frequent three-gene co-occurrence in a representative cohort consisting of 1540 AML patients identified in $6 \%$ of the investigated cases and that co-occurrence of NPM1, FLT3 ITD, and DNMT3A mutations predict a particularly adverse prognosis (8). The same study noticed that deleterious effect of FLT3 ITD was most clinically important in cases with concomitant DNMT3A and NPM1 mutations (8). The frequency of triple NPM1, DNMT3A and FLT3-ITD combined mutations was lower in AML patients from Egypt (1.6\%) (14) than that reported by Papaemmanuil et al (8) for AML cases included in three multicenter clinical trials of the GermanAustrian AML Study Group. Presence of NPM1, DNMT3A and FLT3 ITD triple combined mutations was associated with a short survival in 
Egyptian AML patents (14).

Taking into account that there are few data regarding the NPM1, DNMT3A, and FLT3 combined mutations in AML additional mutation studies are necessary, to determine the appropriate prognosis value of concomitant mutations in AML, especially those focused on investigation of all three genes. Recently, a rare AML case with concomitant four somatic mutations [(namely FLT3 ITD, FLT3 D835 (also known as c. $2504 \mathrm{~A}>\mathrm{T}, \mathrm{D} 835 \mathrm{~V}), D N M T 3 A \mathrm{R} 882 \mathrm{C}$, and NPM1 c.863_864insTCTG)] was reported to be associated with an adverse prognostic and a very short survival (23).

In summary, reviewing the published data, it is not recommended to use FLT3 mutation as a marker for minimal residual disease (MRD) monitoring. FLT3 ITD+ and DNMT3A R882+ double mutations represent an unfavorable prognostic factor in AML patients even after allogeneic hematopoietic stem cell transplantation while DNMT3A and IDH2 mutations frequently co-occured in relapsed or refractory AML cases. The evaluation of FLT3, NPMI and DNMT3A mutational status and routine quantification of mutational burden at diagnosis is therefore an important factor in predicting AML patient outcome. This comprehensive approach will provide data for diagnosis and prognostic significance and will also offer the potential for personalized treatment.

\section{Acknowledgement}

This work was supported by the University of Medicine and Pharmacy of Târgu Mureş, Romania, Research Grant number 15609/13/29.12.2017.

\section{Conflict of interest}

None to declare

\section{Reference}

1. Kim J. Unravelling the genomic landscape of leukemia using NGS techniques: the challenge remains. Blood Res. 2017 Dec;52(4):237-39. DOI: 10.5045/ br.2017.52.4.237

2. Bănescu C, Iancu M, Trifa AP, Dobreanu M, Moldovan VG, Duicu C, et al. Influence of XPC, XPD, XPF, and XPG gene polymorphisms on the risk and the outcome of acute myeloid leukemia in a Romanian population. Tumour Biol. 2016 Jul;37(7):9357-66. DOI: 10.1007/ s13277-016-4815-6

3. Arber DA, Orazi A, Hasserjian R, Thiele J, Borowitz MJ, Le Beau MM, et al. The 2016 revision to the World Health Organization classification of myeloid neoplasms and acute leukemia. Blood. 2016 May 19;127(20):2391405. DOI: 10.1182/blood-2016-03-643544

4. Coriu D, Jardan D, Jardan C, Tălmaci R, Dragomir M, Coliţă A. A new assay to identify recurrent mutations in acute myeloid leukemia using next-generation sequencing. Rev Romana Med Lab. 2014;22(1):93-9. DOI: $10.2478 / \mathrm{rrlm}-2014-0003$

5. Cîrstea M, Coliță A, Ionescu B, Ghiaur A, Vasilescu $\mathrm{D}$, Dobrea $\mathrm{C}$, et al. Therapy-related myelodysplastic syndrome after successful treatment of acute promyelocytic leukemia: case report and literature review. Rev Romana Med Lab. 2017;25(2):165-79. DOI: 10.1515/ rrlm-2017-0013

6. Kumar D, Mehta A, Panigrahi MK, Nath S, Saikia KK. DNMT3A (R882) mutation features and prognostic effect in acute myeloid leukemia in Coexistent with NPM1 and FLT3 mutations. Hematol Oncol Stem Cell Ther. 2018 Jun;11(2):82-9. DOI: 10.1016/j. hemonc.2017.09.004

7. Tang S, Shen H, Mao X, Dai H, Zhu X, Xue S, et al. FLT3-ITD with DNMT3A R882 double mutation is a poor prognostic factor in Chinese patients with acute myeloid leukemia after chemotherapy or allogeneic hematopoietic stem cell transplantation. Int J Hematol. 2017 Oct;106(4):552-61. DOI: 10.1007/s12185-0172256-7

8. Papaemmanuil E, Döhner H, Campbell PJ. Genomic Classification in Acute Myeloid Leukemia. N Engl J Med. 2016 Sep 1;375(9):900-1. DOI: 10.1056/NEJMc1608739

9. Roloff GW, Griffiths EA. When to obtain genomic data in acute myeloid leukemia (AML) and which mutations 
matter. Blood Adv. 2018 Nov 13;2(21):3070-80. DOI: 10.1182/bloodadvances.2018020206

10. Antohe I, Dăscălescu A, Dănăilă C, Zlei M, Ivanov I, Sireteanu A, et al. FLT-3 ITD Positive Acute Basophilic Leukemia with Rare Complex Karyotype Presenting with Acute Respiratory Failure: Case Report. Rev Romana Med Lab. 2018;26(1):87-94. DOI: 10.1515/rrlm2017-0036

11. Döhner H, Estey E, Grimwade D, Amadori S, Appelbaum FR, Büchner T, et al. Diagnosis and management of AML in adults: 2017 ELN recommendations from an international expert panel. Blood. 2017 Jan 26;129(4):424-47. DOI: 10.1182/ blood-2016-08-733196

12. Patnaik MM. The importance of FLT3 mutational analysis in acute myeloid leukemia. Leuk Lymphoma. $2018 \quad$ Oct;59(10):2273-86. DOI: 10.1080/10428194.2017.1399312

13. Bănescu C, Iancu M, Trifa AP, Cândea M, Benedek Lazar E, Moldovan VG, et al. From Six Gene Polymorphisms of the Antioxidant System, Only GPX Pro198Leu and GSTP1 Ile105Val Modulate the Risk of Acute Myeloid Leukemia. Oxid Med Cell Longev. 2016;2016:2536705. DOI: 10.1155/2016/2536705

14. El-Gammal MM, Ebid GT, Madney YM, Abo-Elazm OM, Kelany AK, Torra OS, et al. Clinical effect of combined mutations in DNMT3A, FLT3-ITD and NPM1 among Egyptian acute myeloid leukemia patients. Clin Lymphoma Myeloma Leuk. 2019 Available online 14 February 2019. pii: S2152-2650(19)30007-2, DOI: 10.1016/j.clml.2019.02.001

15. Oltean A, Chincesan MI, Marginean O, Horvath E. Myelodysplastic syndrome with myelofibrosis in a 12-year-old patient - A case report. Rev Romana Med Lab. 2018;26(1):95-103. DOI: 10.1515/rrlm-20170034

16. Patel SS, Kuo FC, Gibson CJ, Steensma DP, Soiffer
RJ, Alyea EP et al. High NPM1-mutant allele burden at diagnosis predicts unfavorable outcomes in de novo AML. Blood. 2018 Jun 21;131(25):2816-25. DOI: 10.1182/blood-2018-01-828467

17. Kunchala P, Kuravi S, Jensen R, McGuirk J, Balusu R. When the good go bad: Mutant NPM1 in acute myeloid leukemia. Blood Rev. 2018 May;32(3):167-83. DOI: 10.1016/j.blre.2017.11.001

18. Moarii M, Papaemmanuil E. Classification and risk assessment in AML: integrating cytogenetics and molecular profiling. Hematology Am Soc Hematol Educ Program. 2017 Dec 8;2017(1):37-44.

19. Grimwade D, Ivey A, Huntly BJ. Molecular landscape of acute myeloid leukemia in younger adults and its clinical relevance. Blood. 2016 Jan 7;127(1):29-41. DOI: 10.1182/blood-2015-07-604496

20. Blau O, Behrenbeck F, Bayanova M, Blau I, Bullinger L. Characteristics of DNMT3A-R882 Mutation in AML. Blood, 2018, 132(Suppl 1):5263.

21. Stein EM, DiNardo CD, Fathi AT, Pollyea DA, Stone RM, Altman JK, et al. Molecular remission and response patterns in patients with mutant-IDH2 acute myeloid leukemia treated with enasidenib. Blood. 2019 Feb 14;133(7):676-87. DOI: 10.1182/ blood-2018-08-869008

22. Ardestani MT, Kazemi A, Chahardouli B, Mohammadi S, Nikbakht M, Rostami S, et al. FLT3-ITD Compared with DNMT3A R882 Mutation Is a More Powerful Independent Inferior Prognostic Factor in Adult Acute Myeloid Leukemia Patients After Allogeneic Hematopoietic Stem Cell Transplantation: A Retrospective Cohort Study. Turk J Haematol. 2018 Aug 3;35(3):158-67.

23. Tripon F, Crauciuc GA, Moldovan VG, Boglis A, Benedek I Jr, Lazar E, et al. Simultaneously FLT3, NPM1 and DNMT3A mutations in adult patients with acute myeloid leukemia- case study. Rev Romana Med Lab. 2019, in press, DOI: 10.2478/rrlm-2019-0022. 